\title{
Using a Residual Gas Analyzer to Monitor Plasma Cleaning of SEM Chambers and Specimens
}

\author{
Ronald Vane ${ }^{1}$ and Michael Cable ${ }^{1}$ \\ 1. XEI Scientific Inc, Redwood City, CA.
}

Residual Gas Analyzers (RGA) are powerful tools to measure and identify gases and contaminants in vacuum chambers. XEI Scientific has manufactured Evactron ${ }^{\circledR}$ plasma cleaners for SEMs since 1999 to remove hydrocarbon contamination. XEI has recently invented a new plasma generation technology with RF external hollow cathode excitation. This new Evactron E50 plasma cleaner operates from a lower base pressure and rapidly removes most hydrocarbons from a vacuum chamber. Because the E50 model operates at lower pressures, RGA spectra can be obtained quickly after the plasma cleaning is stopped. Previous results [1] documented faster pump down times after plasma cleaning with the E50. Fast pump down allows a RGA spectrum to be taken soon after the plasma is stopped so that hydrocarbons can be monitored before being changed by migration.

To use this technique, we installed an ExTorr XT200 RGA and an Evactron E50 De-Contaminator on a FESEM that had never been plasma cleaned. After pump down to base pressure, an initial plasma cleaning was done and a RGA spectrum was taken as shown in Figure 1. A Silicon wafer test specimen was imaged and a black contamination square formed quickly in the scanned area. Imaging was stopped and after 2 minutes of pump down the RGA spectrum shown in Fig. 2 was obtained. This spectrum shows that the interaction of the electron beam with the specimen released hydrocarbon volatiles into the vacuum. A hydrocarbon RGA signature series is shown with peaks every 14 AMU starting at mass 55 . The Evactron E50 was used afterwards for two 5 minute cleans separated with 2 minute pump downs to insure complete cleaning (future studies will be done to find the shortest cleaning end points). This plasma cleaning removed the hydrocarbon signature peaks as seen in Figure 3. This spectrum also revealed some extra peaks that were again identified as Fomblin ${ }^{\circledR}$ fluorocarbon grease [2]. These nonreactive peaks do not create contamination deposits and are benign in in SEMs. The chamber now had a pristine vacuum and no more black square deposition was observed on the specimen which had been left in place. The presence of the non-reactive Fomblin vapors was not seen as a cause of contamination artifacts in this short study, but may deserve more work.

Other experiments will be reported on efforts to combine RGA analysis with Evactron cleaning to determine the end points for plasma cleaning without having to look for deposits or artifacts on specimens with an electron beam. The ability of Evactron E50 to start at high vacuum without extra venting and to allow RGA analysis to be done quickly after the plasma is off makes this obvious procedure useful. The initial procedure is to plasma clean, turn off the plasma, pump down to below $10^{-5}$ Torr, do RGA analysis, resume plasma cleaning if needed, and repeat the RGA process in regular time intervals until clean spectra are obtained. Marker peaks for cleaning include mass $30\left(\mathrm{COH}_{2}\right)$ and 44 $\left(\mathrm{CO}_{2}\right)$

We will also discuss how RGA use can identify leaks and sources of volatiles in SEMs. In this study we found the specimen was the source of most of the contamination in the SEM chamber after imaging. We also confirmed that plasma cleaning does not remove Fomblin fluorocarbon stage lubricant in the 
chamber. Leaks and outgassing can also be studied by RGA, thus RGA studies can confirm a clean and leak-free vacuum chamber.

References:

[1] E. Kosmowska et al, Microsc. Microanal 23 (1) (2017), p. 74.

[2] P. Geltenbort, V.F. Ezhov and V.L. Ryabov, Technical Physics Letters 35 (8) (2009), p. 700.
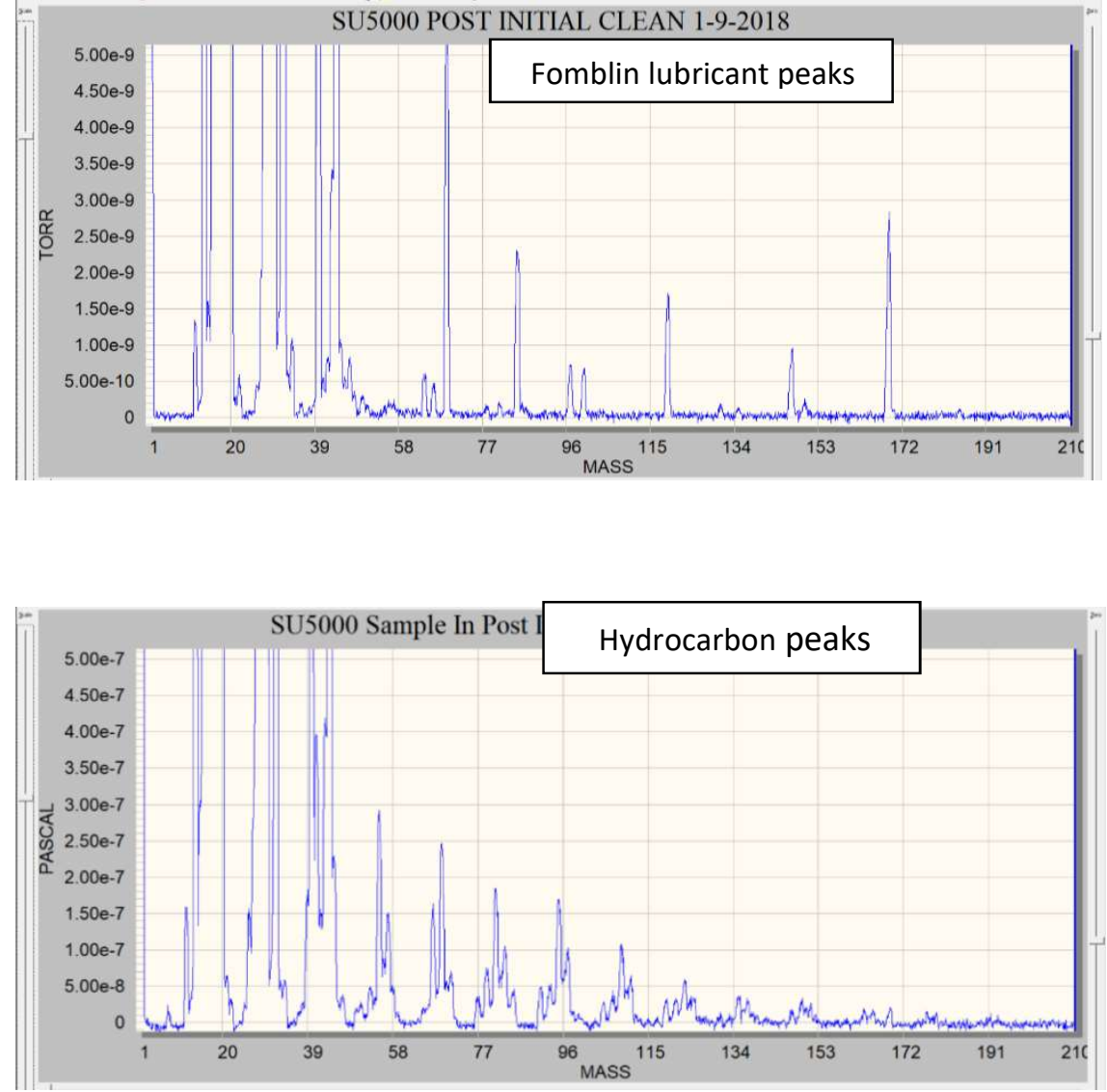

Figure 1. RGA of chamber of a FESEM after venting and plasma cleaning and before imaging. Some residual Fomblin peaks represent very low levels from lubricating stage drives. This level of Fomblin does not cause contamination artifacts.

Figure 2. RGA spectrum showing hydrocarbon signature peaks released by SEM imaging. These cause contamination artifacts.

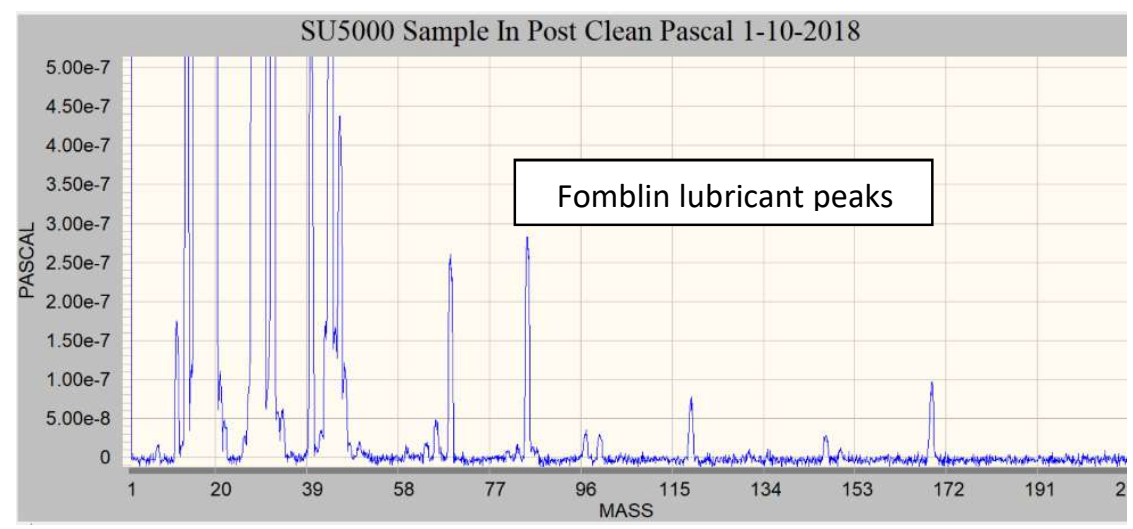

Figure 3. After plasma cleaning again, peaks from Fomblin lubricant are seen but Hydrocarbon peaks disappear. Evactron Oxygen plasma cleaning does not attack the Fomblin fluorocarbon lubricant. 